\title{
On The Design of Cost-efficient and Resilient Hierarchical Backhaul Networks
}

\author{
Ahmed Abdelmoaty*, Diala Naboulsi ${ }^{\ddagger}$, Ghassan Dahman ${ }^{\dagger}$, Gwenael Poitau ${ }^{\dagger}$ and François Gagnon* \\ * Department of Electrical Engineering, École de Technologie Supérieure, Montreal, QC HC3 1K3, Canada \\ \{ahmed.abdelmoaty@lacime.etsmtl.ca\},\{francois.gagnon\}@etsmtl.ca \\ ${ }^{\dagger}$ Ultra Electronics, TCS, Montreal, QC H4T 1V7, Canada \\ \{ghassan.dahman, gwenael.poitau\}@ultra-tcs.com \\ $\ddagger$ Department of Software Engineering and IT, École de Technologie Supérieure, Montreal, QC HC3 1K3, Canada \\ \{diala.naboulsi\}@etsmtl.ca
}

\begin{abstract}
One of the most crucial aspects is modern commercial and tactical wireless networks is the capability of the network's backhaul to forward that massive amount of traffic to the core network. Hence, the careful planning for a resilient and cost-efficient backhaul is compulsory. In this letter, we formulate and solve the problem of hierarchical wireless backhaul design. In our problem, we cover different requirements, namely: link reliability, network resiliency, and low management overhead. We have formulated the problem as Integer Linear optimization Problem (ILP). The results of our simulation in various network scenarios demonstrate the efficiency of our formulation in keeping the backhaul network simple, reliable and resilient. Using practical channel propagation model and nodes density that model wide range of cities, our results show that even with high resiliency requirements, the network traffic can be backhauled with only $5-10 \%$ of the nodes for the considered densities.
\end{abstract}

Index Terms-Hierarchical network topology, Resilient and cost-efficient topology, Wireless backhaul networks.

\section{INTRODUCTION}

$\mathbf{C}$ ellular data traffic witnesses very high growth through the last years. This successive increasing in the traffic demand is not only a result of human activities. The emerging of new types of communications such as video streaming, Device-to-Device (D2D), and machine-type communications which require a suitable accommodation in the mobile network is another factor. Global total mobile data traffic is projected to grow by a factor of around 4.5 from its current value to reach $226 \mathrm{~EB}$ per month in 2026 , the video traffic is account for $77 \%$ of all the traffic [1]. This puts more challenges on the design and planning of mobile networks.

One of the prominent proposed solutions for the ascending behavior of the network traffic is deploying more small cells to absorb the amount of traffic. Consequently, the network will be faced by many challenges as a result of this network's densification. One of these problems is to design a backhaul network that is simple enough to be managed and at the same time it fulfills the reliability and resiliency requirements when backhauling the anticipated huge amount of traffic. Considering these requirements, the design of mobile network's backhaul is one of the challenging tasks for mobile operators, and the summon question is what is the appropriate design for such communication network backhaul?
The backhauls of the communication networks vary depending on the network's objectives and requirements. While wired backhaul networks (e.g., optical fiber (OF) links) provide very reliable solution against weather disruptions and failures, the cost of such links hinders the extended deployment of wired backhaul. On the other hand, wireless backhaul posses many advantages such as cost efficiency, easy deployment, and high capacity.

Despite of the multiple opportunities that wireless backhaul offers, it suffers from link qulaity degradation due to weather related factors. Hence, very specific care is required in designing the topology of wireless backhaul network. In this work, we will consider the hierarchical (i.e., tree) network topology, where it is simple to be managed, and it is flixible such that redundant links can be added to meet the reliability and resiliency requirements [2].

The hierarchical topology consists of a tree with a root node (i.e., Internet gateway) where all the leaves (i.e., base staions (BSs)) are connected to it in a multi-level topology. Mapping this type of topology to the wireless backhaul necessitates the answering of many questions. For example, what is the optimal number of levels at this tree? how many nodes can be included at each level? In each level, how many nodes can be served by each of the nodes at the upper level? What is the role of each node in such topology? Each question of these is related to one or more aspect of the wireless network; the number of levels is connected to the amount of delay; the number of the nodes in each level maps to the network's traffic pattern, and other network aspects such as the reliability and the resiliency might be also mapped. Responds to these questions in the planning phase of the wireless backhaul are very critical. We are aiming to answer some of these questions in our proposed work considering multiple design requirements such as the reliability and resiliency of the network.

The main degradation of the backhaul wireless networks is its vulnerability to the weather disruptions and equipment/site failures. Hence, network resiliency, which is defined as "the ability of a network to defend against and maintain an acceptable level of service in the presence of such challenges" [3], is a critical demand. Among different factors, the resiliency of a network depends on the reliability of its individual links. Availability or connectivity of the network is a critical issue for 
some applications such as vehicular communications, safety and emergency communications, medical and military communications, where the network is required to be connected at whatever quality of service (QoS) level. In other words, the network's connectivity is a first priority.

Several research works investigated the planning of wireless backhaul networks [4]- [7], as will be discussed in the next section. However, to the best of our knowledge, no work has formulated the network topology problem such that it addresses all of the following aspects: a) the network management cost efficiency defined by having simple network structure with the smallest possible number of management nodes i.e., hubs b) the link reliability requirement defined by utilizing only the links that meet a certain reliability metric, and c) the network resiliency defined by having a minimum number of redundant links connecting each BS to the CHs.

The contribution of this letter can be stated in our formulation of the hierarchical backhaul wireless network design problem as an Integer Linear Problem (ILP) model. We propose a simple model, where we optimize the number of required nodes and links for backhauling the traffic. Furthermore, our formulation guarantees all of the above-mentioned network aspects such as link reliability and network resiliency.

The rest of this letter is organized as follows; section II introduces a brief review for the literature related to our work. Section III provides the problem statement and the mathematical formulation of the proposed solution. In section IV, the simulation and the results are detailed. Section V summarizes the findings of this letter.

\section{RELATED WORK}

The planning of a wireless backhaul network topology and the decisions over the nodes and links of the network was discussed in a considerable number of research works [4]- [7]. We are presenting in this section the most relevant research work for our problem formulation and scenario. The decision on the type of the wireless backhaul link was proposed in [4]. The authors introduced a hybrid framework for selecting one of Free Space Optics (FSO), Optical Fibers (OF), or Radio Frequency (RF) links to construct a network topology that maximizes the connectivity of the network. The joint optimization of user association and radio resources in different network topologies has been studied in [6]. The proposed work discussed the effect of two different topologies, namely star and tree topologies in the performance of both the user and the network. On one hand, the star topologies showed a limitation when long links is used. On the other hand, the tree topologies is doing well for a limited number of multihubs between the root and the destination.

Joining the Unmanned Aerial Vehicles (UAVs) as BSs with FSO links has been proposed as a flexible and simple solution of wireless backhauling. The authors in [7] discussed the aspects of link and path reliability to maximize the network resiliency. The work in [5] is considered the closest to our work in this letter. The self-backhauling and the decision on the role of each BS in a hierarchical topology under the resiliency requirement has been studied.

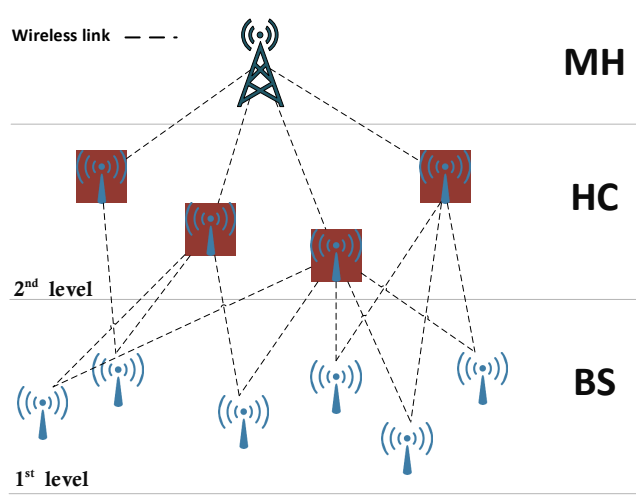

Fig. 1. The considered hierarchical wireless backhaul topology network: the $3^{\text {rd }}$ level contains the Master Hub $(\mathrm{MH})$, the $2^{\text {nd }}$ level contains the Cluster Heads ( $\mathrm{CHs})$, and the $1^{\text {st }}$ level contains the Base Staions (BSs).

In most of the mentioned work, sub-optimal solutions through heuristic algorithms has been proposed as in [7]; other aspects might be missed such as resiliency in [6]. Moreover, the decisions on the role of BSs was not discussed in [4], [6].

We can differentiate our work from the work proposed in [5] with two aspects. The first is that the link reliability was not included there. Furthermore, the network root was not a part of the optimization problem.

\section{System Model AND Problem Formulation}

In this section, we introduce the statement of our research question and we detail the proposed solution as well as the mathematical notations.

\section{A. Problem Statement}

We assume a set of communication nodes which are distributed in a certain topographical area. Based on their locations, the path loss between each node pairs is estimated using a specific channel model. We focus on planning a tree topology wireless backhaul network given the inter-node path loss information. We assume a topology as shown in Fig. 1. In this figure, we consider a network (or sub-network) of 3 tiers. The upper tier (i.e., the $3^{\text {rd }}$ level of the network) is represented by a master hub $(\mathrm{MH})$, which is a node that collects all the traffic from a set of Cluster Heads $(\mathrm{CHs})$ that form the $2^{\text {nd }}$ level of the network. A $\mathrm{CH}$ is a hub node that is responsible for backhauling the traffic from the lower level. Finally, the $1^{\text {st }}$ level of the network consists of a set of Base Stations (BSs).

We aim to design a backhaul topology that is simple, reliable and resilient against propagation impairments, and equipment/site failures. We aim to find the minimum required number of $\mathrm{CHs}$ to backhaul the traffic of the BSs to align with our objective of designing a backhaul network with the simplest possible tree topology. Our task is to decide the level to which each node belongs and its role in the backhaul network. Parallel to these decisions, we need to identify the links among these nodes that will connect the intended tree topology. The resiliency of the designed backhaul network is modeled as k-connectivity links such that we guarantee a 
number of $k$ links between each node at the $1^{\text {st }}$ level and the $\mathrm{CHs}$ at the $2^{\text {nd }}$ level. This is not applicable between the $\mathrm{CHs}$ and the $\mathrm{MH}$, since we are assuming a single $\mathrm{MH}$ node. However, extending our solution to networks with more than two levels and guaranteeing the same level of resiliency for all nodes in the network is left for future work.

To cope with the link reliability requirements, only the links that meet a specific level of reliability from propagation pointof-view are considered to be valid, and consequently, utilized by the network. In this work, we use the Signal-to-NoiseRatio (SNR) as the metric to define the quality of each link. Where, for simplicity at this stage of our work, we assume that interference effect among the different clusters of the network can be mitigated through: a) proper frequency planning which takes advantage of the geographical distribution of the nodes, and b) using directive antennas at the uplink direction of each link (i.e., from the $\mathrm{BSs}$ to the $\mathrm{CHs}$, and from the $\mathrm{CHs}$ to the $\mathrm{MH})$.

\section{B. Mathematical Formulation}

In our model, the backhaul network is modeled as a connected graph. In this graph $G(\mathcal{N}, \mathcal{L}), \mathcal{N}$ is the set of nodes and $\mathcal{L}$ is the set of links between these nodes. The problem is modeled as ILP optimization problem. Four binary decision variables are used, $y_{m}, z_{m}$ where $m \in \mathcal{N}$ to decide about the role of the nodes such that: i) $y_{m}$ is 1 if a node is considered a $\mathrm{CH}$ and 0 if it is a $\mathrm{BS}$, and ii) $z_{m}$ is 1 if the node is a $\mathrm{MH}$, and 0 if it is a $\mathrm{CH}$. Moreover, $x_{(m, n)}, w_{(m, n)}$ have been used to decide if a specific link is included in the backhaul topology in the $1^{\text {st }}$ or the $2^{\text {nd }}$ level of the tree. The notations used for modeling the problem modeling are listed and detailed in Table I.

The objective at (1) is a multi-objective function where our aim is to minimize the cost function. The first term is to minimize the total number of $\mathrm{CHs}$ which is decided by the binary variable $\left(y_{m} \forall m \in \mathbb{N}\right)$. The second term of (1) is to minimize the required links for having a cluster (i.e., number of links at the $2^{\text {nd }}$ level between $\mathrm{CHs}$ and $\mathrm{MH}$ ) which is decided by the binary variable $w_{(m, n)} \forall(m, n) \in \mathcal{L}$.
TABLE I

SYSTEM MODEL NOTATIONS

\begin{tabular}{|c|c|}
\hline Notation & Description \\
\hline \hline $\mathcal{N}$ & $\begin{array}{c}\text { Set of vertices (i.e., nodes), } m, n \text { are elements } \\
\text { in the set }\end{array}$ \\
\hline $\mathcal{L}$ & $\begin{array}{c}\text { Set of edges (i.e., links), the link defined } \\
\text { by it's nodes' indices }\end{array}$ \\
\hline$P_{t}$ & Transmitted power \\
\hline$P_{r-(m, n)}$ & Received power for a link between nodes $m$ and $n$ \\
\hline$P_{r}^{t h}$ & Received power threshold \\
\hline$G_{m}$ & The gain of the antenna at node $m$ \\
\hline$G_{n}$ & The gain of the antenna at node $n$ \\
\hline$\alpha$ & PPP density \\
\hline$P L_{(m, n)}$ & Path loss between node $m$ and node $n$ \\
\hline$f_{c}$ & the carrier frequency \\
\hline $\mathcal{S}$ & Resiliency parameter \\
\hline$x_{(m, n)}$ & $\begin{array}{c}\text { Binary variables }=1 \text { if edge }(m, n) \\
\text { between nodes } m, n \text { is included } \\
\text { at the } 1^{\text {st }} \text { level of the topology }\end{array}$ \\
\hline$w_{(m, n)}$ & $\begin{array}{c}\text { Binary variables }=1 \text { if edge }(m, n) \\
\text { between nodes } m, n \text { is included } \\
\text { at the } 2^{\text {nd }} \text { level of the topology }\end{array}$ \\
\hline$y_{m}$ & Binary variables $=1$ if node $m \in \mathcal{H}$ \\
\hline$z_{m}$ & Binary variables $=1$, if node $m \in \mathcal{M}$ \\
\hline
\end{tabular}

The optimization problem can be stated as Eqs. (1-15).

$$
\begin{array}{ll} 
& \operatorname{minimize}_{w, x, y, z} \sum_{m \in \mathcal{N}} y_{m}+\sum_{(m, n) \in \mathcal{L}} w_{(m, n)} \\
\text { s.t. } \quad & x_{(m, n)} \preceq \quad y_{m}, \quad \forall \quad(m, n) \in \mathcal{L}, \\
& x_{(m, n)} \preceq \quad 1-y_{n}, \quad \forall \quad(m, n) \in \mathcal{L}, \\
& y_{m} \succeq z_{m}, \quad \forall \quad m \in \mathcal{N}, \\
& \sum_{m \in \mathcal{N}} z_{m}=1, \\
& z_{m} \preceq 1-x_{(m, n)}, \quad \forall \quad(m, n) \in \mathcal{L}, \\
& z_{n} \preceq 1-x_{(m, n)}, \quad \forall \quad(m, n) \in \mathcal{L}, \\
& w_{(m, n)}-z_{m} \succeq y_{n}-1, \quad \forall \quad(m, n) \in \mathcal{L}, \\
& w_{(m, n)}-z_{n} \succeq y_{n}-1, \quad \forall \quad(m, n) \in \mathcal{L}, \\
& w_{(m, n)} \preceq \quad 1-x_{(m, n)}, \quad \forall \quad(m, n) \in \mathcal{L}, \quad \\
& \sum_{n \in \mathcal{N}} x_{(m, n)} \succeq \mathcal{S} *\left(1-y_{m}\right), \quad \forall \quad m \in \mathcal{N} \\
& \left(P_{r-(m, n)}-P_{r}^{t h}\right) * x_{(m, n)} \succeq 0, \forall \quad(m, n) \in \mathcal{L} \\
& \left(P_{r-(m, n)}-P_{r}^{t h}\right) * w_{(m, n)} \succeq 0, \forall \quad(m, n) \in \mathcal{L} \\
& x_{(m, n)}, w_{(m, n)} \in\{0,1\}, \quad \forall \quad(m, n) \in \mathcal{L} \quad \\
& y_{m}, z_{m} \quad \in\{0,1\}, \quad \forall \quad m \in \mathcal{N}
\end{array}
$$

Constraints $(2,3)$ ensure that each link at the $1^{\text {st }}$ level will be between a $\mathrm{CH}$ and a BS. The constraints work as if a certain link has been selected in the $1^{\text {st }}$ level of the topology (i.e., $\left.x_{(m, n)}=1\right)$, then one node e.g., $m$ should be selected as a $\mathrm{CH}$ and the other node $n$ will be considered a BS. 
Constraint (4) is used to ensure that the root node (i.e., $\left.z_{m}=1\right)$ will be a member of the CHs family $\left(y_{m}=1\right)$. We are assuming one root for this sub-network or network; hence, the sum of $z_{m}$ should be equal to 1 which has been guaranteed by constraint (5).

Constraints $(6-10)$ are used to organize the relations between nodes and links in the two levels of the topology. Eq. $(6,7)$ ensure that whenever a node is selected as a $\mathrm{MH}$ $\left(z_{m}\right.$ or $\left.z_{n}=1\right)$, it will not be connected to a node at the $1^{\text {st }}$ level (i.e., $x_{(m . n)}=0$ ).

Constraints $(8,9)$ are doing the same at the $2^{\text {nd }}$ level of the topology. Whenever a node is selected as a BS, i.e., $y_{n}=1$, it is forbidden to be connected to one of the nodes or links of the $2^{\text {nd }}$ level $\left(z_{m}\right.$ or $z_{n}$, and $\left.w_{(m, n)}\right)$ respectively. Finally, Eq. (10) ensures that the optimizer will not activate the same link for both levels at the same time, $x_{(m, n)}$ can not be equal to 1 when $w_{(m, n)}$ is active.

Constraint (11) ensures the resiliency of the network by forcing $\mathcal{S}$-connectivity between each $\mathrm{BS}$ and $\mathrm{CH}$, in this constraint; we are forcing that if a node $m$ is not selected as a $\mathrm{CH}$, then there must be at least $\mathcal{S}$ links between $m$ and it's neighbors.

Constraints $(12,13)$ ensure the link quality at each tier of the network by maintaining the received power of each active link $\left(x_{(m, n)}, w_{(m, n)}\right)$ is greater than a certain threshold i.e., $P_{r}^{t h}$. Finally, eqs. $(14,15)$ define the nature of the four decision variables.

\section{Simulation AND Results}

In our simulation case, we focus on a scenario of Rapidly Deployable Network (RDN) for tactical backhaul of an isolated area or where the commercial network is unavailable. In our subnetwork, the nodes are distributed according to Matern Hard-Core process (MHCP) type II with minimum distance $R$. MHCP is a Poisson Point Process (PPP) that maintains a minimum distances between the nodes and it is a common process for modeling wireless networks [8], [9]. We consider different nodes distribution densities that vary from $1 \mathrm{BS} / \mathrm{KM}^{2}$ to $10 \mathrm{BS} / \mathrm{KM}^{2}$.

We use the WINNER-II B5a (i.e., rooftops to rooftops) model to estimate the inter-node path loss [10]. Furthermore, a practical antenna gains for two different frequencies are considered. For each link, we assume the node at the higher layer to be provided with an Omni antenna, i.e., the MH uses an Omni antenna to communicate with the $\mathrm{CHs}$, and the $\mathrm{CHs}$ use Omni antennas to communicate with their BSs. On the other hand, for each link, we assume the node at the lower layer to be provided with a directive antenna, i.e., the CHs use directive antennas to communicate with the $\mathrm{MH}$, and the $\mathrm{BSs}$ use directive antennas to communicate with the CHs [11]. The simulation parameters are summarized in Table II.

The network designer could be more conservative against equipment/site failures and increase the required resiliency level by selecting a large number of support links between each $\mathrm{BS}$ and the $\mathrm{CHs}$ (i.e., $\mathcal{S}>1$ ). As shown in Fig.2-a, where the ratio between the utilized $\mathrm{CHs}$ and the set of all nodes is shown in the left axis, and the number of utilized

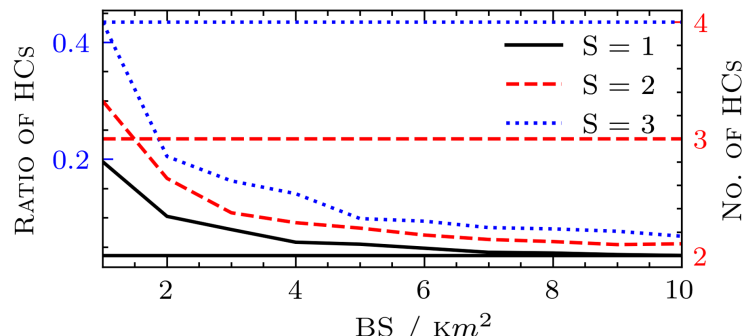

(a)

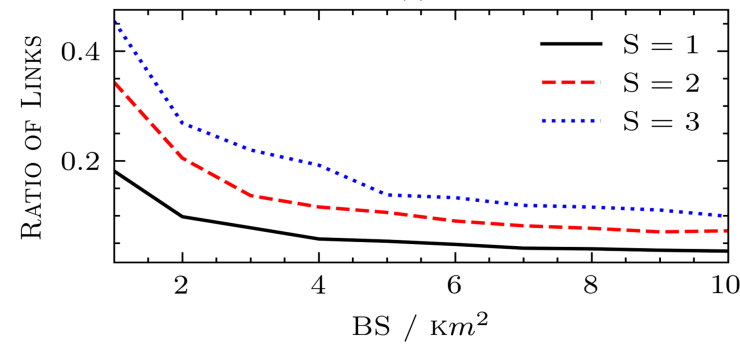

(b)

Fig. 2. Ratio of utilized Links and CHs for different resiliency levels. (a) Left-axis: Ratio of CHs (i.e., no. of utilized CHs / no of all nodes). Rightaxis: No. of CHs (no. of utilized CHs). (b) Ratio of utilized links (no. of utilized links / No. of all available links).

CHs is shown in the right axis for different densities of the network nodes. As it is clear; the larger the value of $S$ (i.e., restrictive resiliency requirements), the larger the number of utilized CHs. The same trend is shown in Fig.2-b where the ratio between utilized links in the topology and the set of available links is presented. In specific, The ratio of both $\mathrm{CHs}$ and links is considerably high when the network is less dense and reaches almost 20\% - 45\%. However, when the network is getting denser, this ratio is dropped down to approaches less than $10 \%$ when $\mathcal{S}$ is set to 2 . This reflects the strength of our formulation in keeping the network simple and reducing the management cost significantly even when the density of the network is increased which is an expected trend for the next generation networks.

The answer of our research question of this letter: How to obtain a reliable, resilient, and simple wireless backhaul topology to connect a set of communication nodes? is very dependent on the propagation environment. Hence, Figs. 3, and 4 are devoted to demonstrate the performance of our formulation in different propagation scenarios.

In Fig.3, we are comparing the performance of our model at two different frequencies $(2$ and $5 \mathrm{GHz}$ ). The ratio of the utilized $\mathrm{CHs}$ and the utilized links to the set of nodes/available links, respectively is shown. It's very apparent that the ratio of the $\mathrm{CHs}$ is the same in the two scenarios. The reason behind this is that our formulation is forcing the solver to find the minimum possible number of CHs to backhaul the network's traffic which is a total of three $\mathrm{CHs}$ (one $\mathrm{MH}$ i.e., Eq. (5) + two $\mathrm{CHs}$ to satisfy the resiliency requirements for $\mathcal{S}=2$ i.e., Eq. (11)). The effect of having higher path loss at $5 \mathrm{GHz}$ compared to $2 \mathrm{GHz}$ is (almost) compensated for by the higher antenna gain at the higher frequency.

From our previous analysis it is clear that the number of 


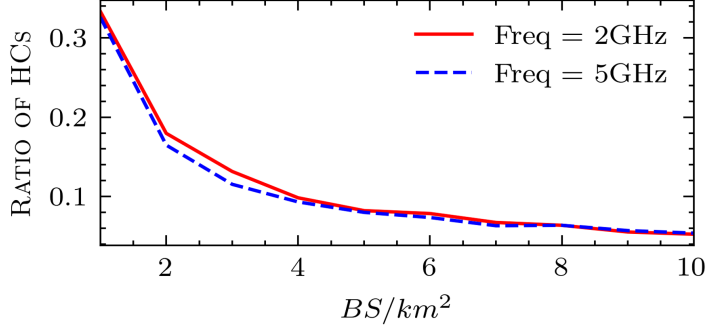

(a)

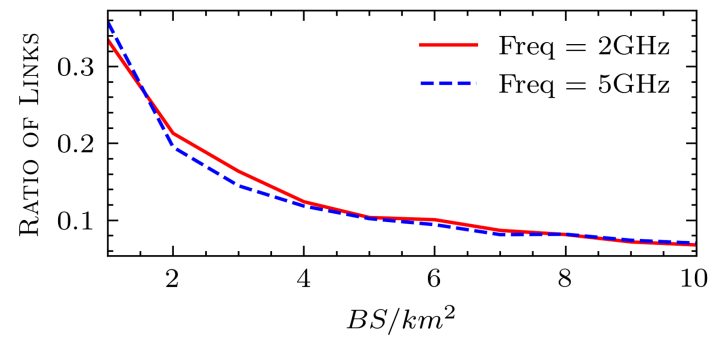

(b)

Fig. 3. Ratio of CHs (a) and ratio of links (b) for two operating frequencies

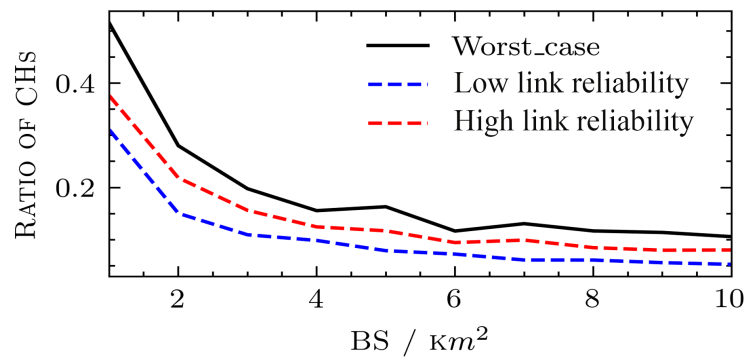

(a)

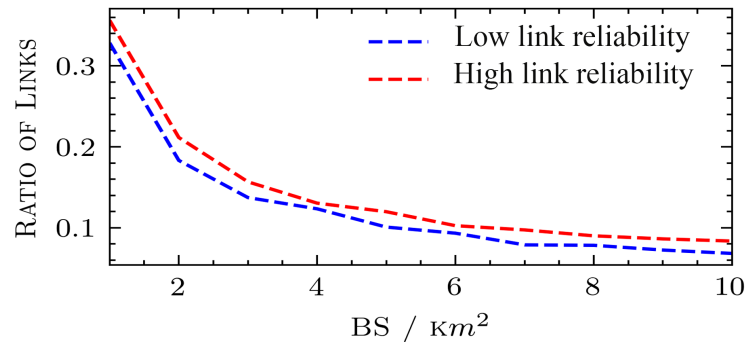

(b)

Fig. 4. Ratio of $\mathrm{CHs}$ (a) and ratio of links (b) for different link reliability requirements

the available links for connecting our backhaul topology is a prominent factor of the decision of the number of required CHs. Fig. 4 presents the performance of our formulation in the presence of more restricted reliability threshold. In the cases demonstrated in Figs. 2 and 3, a link is considered to be valid if it has a minimum SNR of $10 \mathrm{~dB}$, where no fade margin against weather disruptions is accounted for. However, here we compare two cases: a) low-reliability case, where no fade margin against weather disruptions is considered. In this case, each link, by definition, will meet the reliability requirements only $50 \%$ of the time. b) high-reliability case, where a 20 $\mathrm{dB}$ fade margin against weather disruptions is accommodated for when calculating the link budget. Based on the Olsen-
TABLE II

SIMULATION PARAMETERS

\begin{tabular}{|c|c|}
\hline Parameter & Value \\
\hline \hline Simulation area & $10 \mathrm{~km}^{2}$ \\
\hline Operating frequency & $2 \mathrm{GHz}$ and $5 \mathrm{GHz}$ \\
\hline Transmitted Power & $20 \mathrm{dBm}$ \\
\hline Omni-antenna gain & $6 \mathrm{~dB}($ at $2 \mathrm{GHz})$ and $8 \mathrm{~dB}($ at $2 \mathrm{GHz})$ \\
\hline Parabolic antenna gain & $20 \mathrm{~dB}($ at $2 \mathrm{GHz})$ and $28 \mathrm{~dB}($ at $2 \mathrm{GHz})$ \\
\hline Node densities & $1: 10 \mathrm{BS} / \mathrm{KM}^{2}$ \\
\hline Minimum inter-node distance & $200 \mathrm{~m}$ \\
\hline
\end{tabular}

Segal model and our simulation parameters, this fade margin guarantees a link reliability of $99.99 \%$ [12].

Fig.4-a shows the ratio of $\mathrm{CHs}$ at the low-reliability and high-reliability cases (averaged over 20 simulation runs). It is obvious that the higher the reliability requirement, the more the required $\mathrm{CHs}$. Moreover, the ratio of $\mathrm{CHs}$ at the high-reliability obtained from the worst simulation run (i.e., the ratio of the maximum number of hubs to the average number of nodes) is shown in solid black in Fig.4-a, where only $10 \%$ of the nodes are required to backhaul the traffic at dense networks.

\section{CONCLUSION}

In this letter, we have proposed a formulation for the design of a hierarchical wireless backhaul topology that respects the network requirements including reliability, resiliency, and low management overhead. We have formulated the problem as Integer Linear optimization Problem (ILP). The results of our simulation in variant network scenarios demonstrate the efficiency of our formulation in keeping the backhaul network simple, reliable and resilient. Our results showed that even with high resiliency requirements, the network traffic can be backhauled with only $5-10 \%$ of the nodes. Moreover, with different scenarios of propagation environment; our formulation was maintained to deliver the same performance.

\section{REFERENCES}

[1] Ericsson Mobility Report. 2020. [Online]. Available: $\quad$ https://www.ericsson.com/4adc87/assets/local/mobilityreport/documents/2020/november-2020-ericsson-mobility-report.pdf.

[2] M. Jaber, F. J. Lopez-Martinez, M. A. Imran, A. Sutton, A. Tukmanov, and R. Tafazolli, "Wireless backhaul: Performance modeling and impact on user association for 5G," IEEE Transactions on Wireless Communications, vol. 17, no. 5, pp. 3095-3110, 2018.

[3] D. Hutchison and J. P. Sterbenz, "Architecture and design for resilient networked systems," Computer Communications, vol. 131, pp. 13-21, 2018.

[4] M. H. Al-mekhlafi, A. S. Ibrahim, Y. A. Fahmy, and M. M. Khairy, "Resilient hybrid optical-RF backhauling for tiered networks," Physical Communication, vol. 36, p. 100814, 2019.

[5] Y.-H. Chiang and W. Liao, "mw-HierBack: A cost-effective and robust millimeter wave hierarchical backhaul solution for HetNets," IEEE Transactions on Mobile Computing, vol. 16, no. 12, pp. 3445-3458, 2017.

[6] N. Sapountzis, T. Spyropoulos, N. Nikaein, and U. Salim, "Optimal downlink and uplink user association in backhaul-limited HetNets," in IEEE INFOCOM 2016-The 35th Annual IEEE International Conference on Computer Communications. IEEE, 2016, pp. 1-9.

[7] Z. Gu, J. Zhang, and Y. Ji, "Topology optimizing in FSO-based UAVs relay networks for resilience enhancement," Mobile Networks and Applications, vol. 25, no. 1, pp. 350-362, 2020. 
[8] B. Matérn, Spatial variation. Springer Science \& Business Media, 2013, vol. 36

[9] U. Schilcher, J. F. Schmidt, and C. Bettstetter, "On interference dynamics in matern networks," IEEE Transactions on Mobile Computing, vol. 19, no. 7, pp. 1677-1688, 2019.

[10] J. Meinilä, P. Kyösti, T. Jämsä, and L. Hentilä, "Winner II channel models," Radio Technologies and Concepts for IMT-Advanced, pp. 3992, 2009.

[11] R. Taori and A. Sridharan, "Point-to-multipoint in-band mmwave backhaul for 5G networks," IEEE Communications Magazine, vol. 53, no. 1, pp. 195-201, 2015.

[12] R. Olsen and B. Segal, "New techniques for predicting the multipath fading distribution on vhf/uhf/shf terrestrial line-of-sight links in canada," Canadian Journal of Electrical and Computer Engineering, vol. 17, no. 1, pp. 11-23, 1992. 